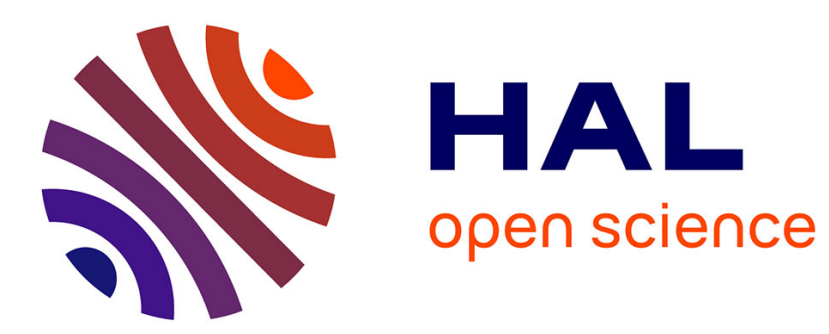

\title{
Bistable behaviour and thermo-acoustic instability triggering in a gas turbine model combustor
}

\author{
Antoine Renaud, Sebastien Ducruix, Laurent Zimmer
}

\section{To cite this version:}

Antoine Renaud, Sebastien Ducruix, Laurent Zimmer. Bistable behaviour and thermo-acoustic instability triggering in a gas turbine model combustor. Proceedings of the Combustion Institute, 2017, 36 (3), pp.3899-3906. 10.1016/j.proci.2016.08.007 . hal-01424851

\section{HAL Id: hal-01424851 \\ https://hal.science/hal-01424851}

Submitted on 28 Feb 2020

HAL is a multi-disciplinary open access archive for the deposit and dissemination of scientific research documents, whether they are published or not. The documents may come from teaching and research institutions in France or abroad, or from public or private research centers.
L'archive ouverte pluridisciplinaire HAL, est destinée au dépôt et à la diffusion de documents scientifiques de niveau recherche, publiés ou non, émanant des établissements d'enseignement et de recherche français ou étrangers, des laboratoires publics ou privés. 


\title{
Bistable behaviour and thermo-acoustic instability triggering in a gas turbine model combustor
}

\author{
Antoine RENAUD ${ }^{\mathrm{a}, *}$, Sébastien DUCRUIX ${ }^{\mathrm{a}}$, Laurent ZIMMER ${ }^{\mathrm{a}}$ \\ ${ }^{a}$ Laboratoire EM2C, CNRS, CentraleSupélec, Université Paris-Saclay, Grande Voie des Vignes, 92295 Châtenay-Malabry cedex, FRANCE
}

\begin{abstract}
In a liquid-fueled two-stage gas turbine model combustor, a bistable point where two different flame states can exist is studied in non-reacting and reacting conditions with the help of fast spray-PIV and chemiluminescence imaging. The first flame, named $s V$, is stabilized thanks to an internal reaction zone that modifies the air flow compared to non-reacting conditions. The second state, associated with a lifted flame, exhibits a strong thermo-acoustic instability linked to the quarter-wave mode of the chamber and piloted by a varying liquid-fuel supply to the flame. Using wellcontrolled air flow rate modulations, the switch from the quiet $s V$ state to the lifted one is studied in a time-resolved fashion. The analysis shows that the sensitivity of the $s V$ state to perturbations is responsible for the change in flame shape which then triggers the onset of the thermo-acoustic instability.
\end{abstract}

Keywords:

Flame dynamics, bifurcation, liquid fuel, staging control, precessing vortex core

\section{Introduction}

Lean Premixed Prevaporized (LPP) combustion is an interesting candidate to replace diffusion flames in aeronautical gas turbines in order to reduce pollutant emissions. By supplying a relatively uniform lean mixture of fuel and air, the goal is to reduce flame temperature and thus the creation of thermal NOx. Such regimes are however often faced with stability issues such as uncontrolled dynamics, flashback or blowout $[1,2]$. In particular, thermo-acoustic instabilities can occur from the coupling between acoustic oscillations and heat release [3]. Staged injectors can help tackle this issue by injecting a small amount of fuel upstream to create a piloting region that stabilizes the system. They can be associated with a multipoint injection device for the main stage in order to promote a fast and efficient mixing and thus get closer to LPP requirements for most of the power generation. The dynamics of multipoint staged injector as well as the effects of staging remain yet to be fully understood. Indeed, numerical simulations [4] as well as experimental studies [5] show that the flame shape and

${ }^{*}$ Corresponding author: Laboratoire EM2C, Grande Voie des Vignes, 92295 Châtenay-Malabry cedex, FRANCE

Email address: antoine.renaud@centralesupelec.fr (Antoine RENAUD)

Preprint submitted to Proceedings of the Combustion Institute behaviour is highly dependent on the burner history and on potential external perturbations.

The purpose of this paper is to complete the study performed in [6], which showed a naturally occurring transition between two states. Focusing on a fixed staging operating point that shows a bistable behaviour with completely different flame and air flow structures, stabilization mechanisms are presented and an artificially triggered transition from one state to the other is described.

\section{Experimental setup and diagnostics}

\subsection{Experimental setup}

The BIMER experimental device [6] revolves around a model of an industrial staged injector placed in a plenum before a $500 \mathrm{~mm}$-long optically-accessible water-cooled combustion chamber with a square section $(150 \mathrm{~mm})$. The chosen fuel is dodecane and, to enhance vaporization, air flows through an electric preheater (Sylvania SureHeat Max) before entering the plenum. Details of the injector can be seen on the horizontal cut shown in Fig. 1.

The injector is composed of two stages each including its own air swirler and fuel injection. Both swirlers are co-rotating and the air flow, regulated by a mass flow

December 3, 2015 


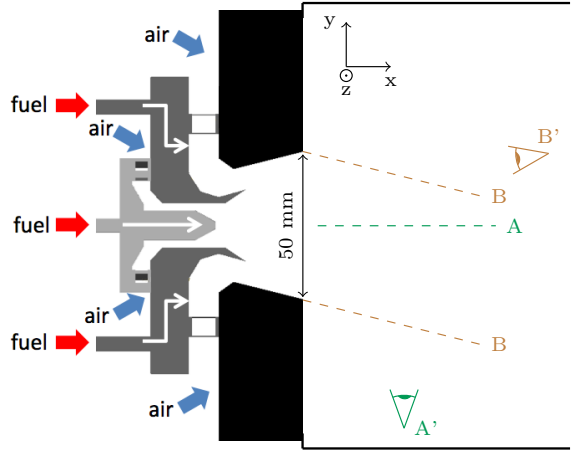

Figure 1: Top view of the injection device positioned at the entrance of the combustion chamber, showing the two injection stages (pilot: light gray, take-off: dark gray). A laser sheet (A) illuminates the (x,z) plane in the center of the chamber to allow the observation (A') of the fuel droplets Mie scattering signal. An expanded laser beam (B) is sent in the injector hole to visualize (B') the fuel spray near the pilot fuel nozzle.

controller (Bronkhorst In-Flow), is divided between the stages by the fixed geometrical design of the injector.

The upstream stage, called the pilot stage (light gray in Fig. 1), allows $13.5 \%$ of the air mass to flow through and is equipped with an 18-vane swirler with a geometrical swirl number close to 0.6. The fuel injection is made with a pressurized nozzle (MMP 6390-0-0-03) that creates a hollow cone of droplets. The take-off stage (downstream, dark gray), allows $86.5 \%$ of the air to flow through its 20 -vane swirler with a geometrical swirl number around 1 . The liquid fuel is injected through a multipoint device made by ten $0.3 \mathrm{~mm}$ holes placed in the cross flow of every other swirler vane to ensure proper atomization. A staging parameter is defined to characterize the fuel distribution in the injector. It corresponds to the ratio between the amount of fuel injected through the pilot stage and the total amount of fuel.

In order to perform controlled air flow rate modulations, a siren-like device (CBOne, Graz, Austria) is placed before the plenum. It works by rotating a toothed wheel in front of a nozzle through which the air flows. The rotational speed of the wheel controls the modulation frequency. The amplitude of the fluctuations are independently modified by changing the relative positions of the wheel and the nozzle. A thorough description of the system can be found in [7].

In this study, the chosen operating point corresponds to an air flow rate of $43 \mathrm{~g} / \mathrm{s}$ (preheated at $433 \mathrm{~K}$ ) and a global equivalence ratio of 0.6 . The thermal power generated by the combustion is around $72 \mathrm{~kW}$. A similar operating point is presented in [6] but, in the present case, the staging parameter is fixed at $15 \%$, meaning that $15 \%$ of the fuel is injected through the pilot nozzle and the remaining $85 \%$ through the multipoint device. This takeoff oriented injection represents the expected operation of a staged multipoint injector when high power with low pollutant emissions is needed.

\subsection{Diagnostics}

The fuel spray inside the chamber can be observed thanks to the Mie scattering signal of the droplets crossing a laser sheet. The associated setup is schematically shown as (A-A') in Fig. 1. The light sheet is generated by two Nd:YAG lasers working at $10 \mathrm{kHz}$ each with a delay of $20 \mu$ s between them. A high-speed camera (Photron Fastcam SA-5) equipped with a $50 \mathrm{~mm}$, $\mathrm{f}: 1.4$ lens (Nikon) and a 532( \pm 10$) \mathrm{nm}$ filter records the light scattered by the droplets. The $704 \times 520$ pixels sensor size enables the recording of an equivalent $176 \times 130 \mathrm{~mm}$ region. Each recording can last up to $1.56 \mathrm{~s}$, leading to 31274 recorded images.

Thanks to the tunable delay between the lasers, it is possible to perform a Particle Image Velocimetry (PIV) processing on two consecutive fuel spray images. Because the Mie scattering signal from the droplets is used, the seeding is highly non-uniform and the resulting velocity field does not necessarily represents the underlying air flow. In the present study, the images are treated with the open source PIVLab software, using a direct cross-correlation algorithm on $20 \times 20$ pixels interrogation windows with $60 \%$ overlap.

Because what happens upstream of the opticallyaccessible combustion chamber can provide interesting information, a visualization technique aiming at observing the spray near the pilot nozzle is also developed in the present study, schematically represented as (B-B') in Fig. 1. It consists in sending an expanded laser beam $(\mathrm{Nd}: Y A G, 10 \mathrm{kHz}$ ) into the hole linking the chamber and the injector and recording the Mie scattering signal from the early droplets with a high-speed camera (Photron Fastcam SA-5) equipped with a $105 \mathrm{~mm}$, f:5.6 lens (Nikon) and a 532( \pm 10$) \mathrm{nm}$ filter. Because of the volume lighting, quantitative spatial information cannot be extracted from such images but the high speed allows for the temporal resolution of some phenomena.

To monitor the flame in the reacting cases, $\mathrm{CH}^{*}$ chemiluminescence is recorded with a high speed camera (Photron Fastcam SA-X) equipped with a light intensifier (Hamamatsu C10880-03C), a $50 \mathrm{~mm}$ f:1.4 lens and a $431( \pm 10) \mathrm{nm}$ filter. The $512 \times 512$ pixels images enable the recording of a $153 \times 153 \mathrm{~mm}$ region in the beginning of the chamber. The orientation of the camera is the same as A' in Fig. 1 and the acquisition frequency is $10,000 \mathrm{fps}$. 
Simultaneously to all the previously described optical measurements, the pressure fluctuations in the chamber are recorded with a B\&K microphone (4938) placed in a home-built water-cooled semi-infinite waveguide located at half the length of the chamber. Its signal is acquired with a NI acquisition card (PCI-MIO-16E-4) at a $32768 \mathrm{~Hz}$ frequency.

\section{Non-reacting conditions}

Before analyzing the response of the burner in reacting conditions, it is first needed to observe its nonreacting behaviour to provide a reference point. Air is still preheated at $433 \mathrm{~K}$ but no combustion reaction occurs. Key information are contained in the mean shape of the non-reacting fuel spray, shown as a black contour on the image on the left of Fig. 2.

The fuel spray contours are obtained from the Mie scattering recordings inside the combustion chamber. Because the signal is created by relatively dilute small droplets, what matters is the fluctuations of the pixel values over the recording legnth rather than their average intensity which would only highlight background features. The contours shown in Fig. 2 are thus based on the Root Mean Square (RMS) image created with the RMS value for each individual pixel. The black line follows a threshold set at $15 \%$ of the maximum RMS value.

The average spray is quite wide which probably highlights a good mixing between the fuel and the air. Still, the corners and the center of the chamber do not show a large number of fuel droplets. Phase Doppler Anemometry (PDA) measurements performed for this operating point enable to compute the Sauter Mean Diameter for the spray, which is below $15 \mu \mathrm{m}$. This value is low enough to consider that the majority of the droplets in the spray have a relatively low Stokes number and are thus good tracers of the air flow. The conditional PIV treatment of the Mie scattering images can then be used to analyse the gaseous flow.

Because of the relative scarcity of the fuel droplets, only a limited number of valid vectors can be obtained for each image pair. To avoid misinterpretations due to non-converged statistics when computing average fields, a threshold is set to dump the data at the spatial positions where there are not enough valid vectors. This means that at least 300 valid vectors were required to compute any value shown in Fig. 2.

On the left of Fig. 2, one can observe the average velocity fields for the non-reacting case. A jet region, characterized by positive velocity values can clearly be identified, surrounded by two reverse velocity regions.
This structure is expected since it comes from the vortex breakdown due to the highly swirling flow in a confined environment. It creates a swirling jet surrounding an Inner Recirculation Zone (IRZ) and surrounded by an Outer Recirculation Zone (ORZ).

The bottom part of the image shows that the vertical velocity is close to zero in most of the chamber. Two regions are characterized by positive vertical velocity values a bit above $5 \mathrm{~m} / \mathrm{s}$, showing a converging behaviour of the liquid fuel. When compared with oilseeded cold flow measurements (not shown here), these regions seem to be responsible for feeding the upstream part of the IRZ which appears in white here because the smaller fuel droplets trapped there vaporize before giving any exploitable velocity value.

From a dynamical point of view, analyses of the Mie scattering fields show the presence of a coherent structure that creates a helical-like modulation inside the spray. Studies of this phenomenon [5] have shown that it corresponds to the trace of a Precessing Vortex Core (PVC) that has roots near the pilot nozzle. Even though all air velocity fluctuations associated with the PVC seem to be damped once in the chamber, it causes a slightly varying repartition of the liquid fuel that can still be observed. In order to monitor the PVC at its origin, internal Mie scattering visualizations are used (B-B' in Fig. 1). They enable to confirm the precessing nature of the phenomenon and, thanks to a small averaging window, to obtain a signal that contains the precession frequency of the structure. In the present non-reacting case, the PVC frequency is measured at $1950 \pm 20 \mathrm{~Hz}$. This information on the state of the flow before the combustion chamber gives precious insight on what is happening in non commonly observed areas.

\section{Reacting conditions}

In reacting conditions, two different flame shapes can be observed, depending on the history of the burner. Details on this phenomenon can be found in [6].

\section{1. sV flame}

The burner is ignited at a low power, pilot-only operating point. The flame then takes a V shape. Power is then increased and staging decreased to reach the present operating point. The flame topology changes during this operation and the flame shows a shape that is called $s V$. By averaging images of $\mathrm{CH}^{*}$ chemiluminescence and carrying out Abel deconvolution, the flame shape is obtained (top of Fig. 3).

The reaction zone is not strongly marked and is globally diverging away from the chamber axis. At the top 

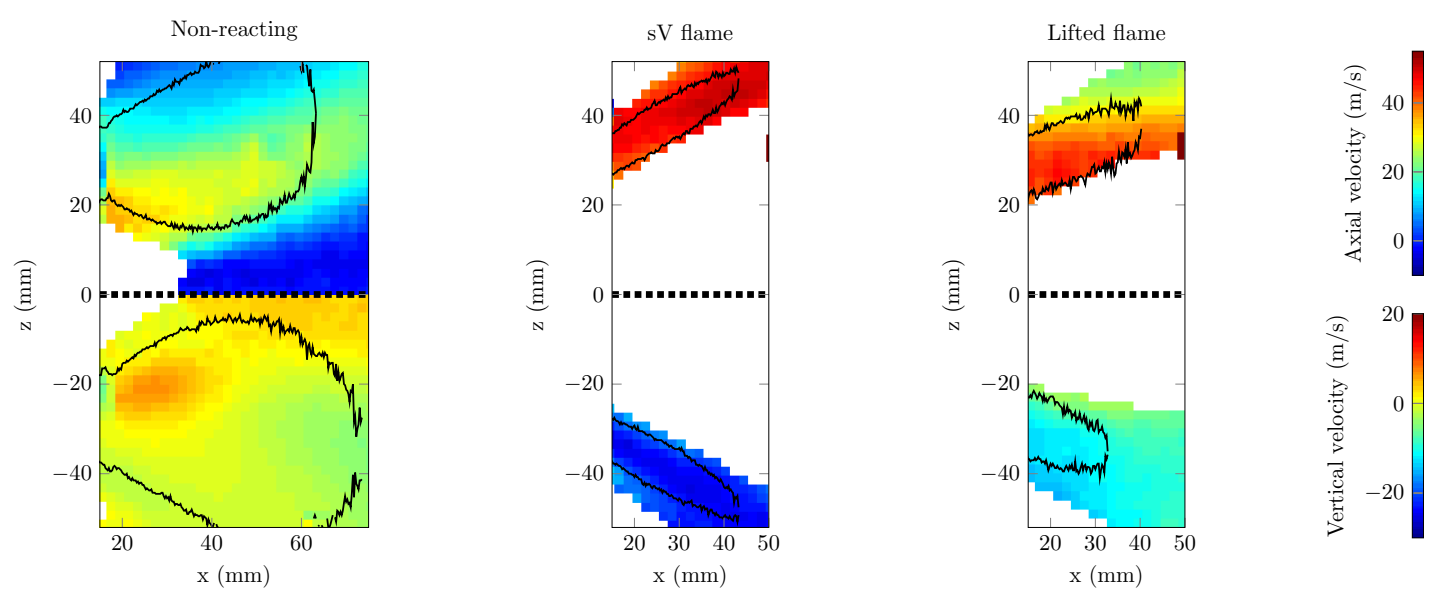

Figure 2: Spray contour (black line) and axial (top) and vertical (bottom) average velocity fields. The dotted line represents the axis of the chamber which is also an axis of symmetry. The white areas correspond to a number of valid vectors below 300 , indicating a poor concentration of droplets. The origin of the axes is at the center of the junction between the injector and the chamber.

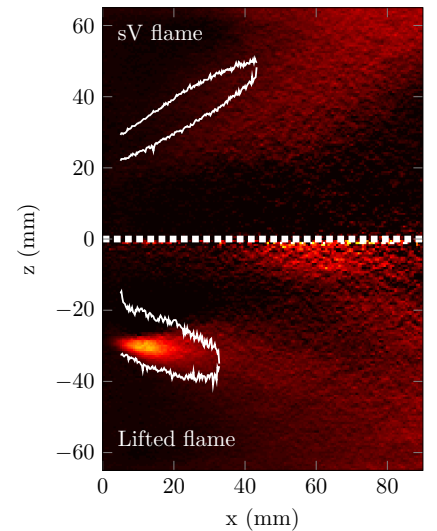

Figure 3: Abel deconvoluted average $\mathrm{CH}^{*}$ chemiluminescence images of the $s V$ flame (top) and the lifted flame (bottom). The white lines represent the average spray shape in each case.

border of the image, it seems to progress slightly upstream, thus showing an interaction with the chamber walls. Looking at its position relatively to the droplets (shown in white), it seems that the flame is surrounded by the liquid fuel spray. The shape of the latter seems different from what is observed in non-reacting conditions, being much thinner and diverging. It is thus interesting to look at the associated velocity fields represented in the middle of Fig. 2.

Two main phenomena are acting on the spray shape. The droplets are transported at a certain position by the air flow and they have to remain liquid in the process. If the difference between the non-reacting case and the $s V$ case lay only in additional heat release from the flame enhancing vaporization, the velocity fields would look similar in the regions where the non-reacting and $s V$ spray overlap. Figure 2 shows that it is not the case, the flame is thus responsible for a change in the air flow as well. Indeed, the axial velocity field shows an almost constant $50 \mathrm{~m} / \mathrm{s}$ value while the vertical velocity field highlights a strongly diverging trajectory with velocity values around $-30 \mathrm{~m} / \mathrm{s}$.

Further information on the $s V$ state can be obtained using the results of the internal visualizations to monitor the PVC behaviour. In this case, the PVC frequency is measured in the range $2020 \pm 10 \mathrm{~Hz}$ which is both higher on average and narrower in fluctuation than in the nonreacting case. Such a change must be related to a modification of the air flow inside the injector itself and other observations lead to associate this with the existence of an internal flame anchored on the pilot nozzle that cannot be detected from the combustion chamber.

The $s V$ flame is relatively quiet with a moderate sound pressure level (around $136 \mathrm{~dB}$ ) in the chamber and no specific frequency content (broadband noise). The use of a dominant multipoint injection also leads to smaller droplets and an expected proper mixing. In this regard, it seems that the $s V$ state fulfills the industrial criteria for a main LPP flame stabilized by a smaller pilot flame. In some cases however, the present operating point can show another kind of flame: the lifted flame.

\subsection{Lifted flame}

The lifted flame is generally observed when the staging goes to a multipoint-only injection and is increased back to $15 \%$ of pilot [6]. In the last part of the present study, another way of switching from the $s V$ to the lifted state using siren-induced air flow rate modulations is also presented. 
The average flame shape for the lifted flame can be seen at the bottom of Fig. 3. The reaction zone seems mostly spread in the downstream part of the region of interest. Some signal can be observed upstream but it does not originate from the inside of the injector. This leads to believe that the flame is completely detached and thus aerodynamically stabilized by the swirling flow. The spray looks quite thick and surrounded by the flame.

The axial and vertical average velocity fields associated with the lifted state can be observed on the right of Fig. 2. The shape of the fields is drastically changed when compared with the $s V$ flame but seems rather close to the non-reacting case. The main difference between these two cases lies mainly in the higher velocities observed for the lifted state. This increase can be explained by the conservation of the mass flow rate: the presence of the flame heats the air up, thus reducing its density. The smaller spread of the spray can as well be explained by this temperature increase that then enhances fuel vaporization.

The internal visualizations show that the PVC is still present, although less marked than for the $\mathrm{sV}$ flame. The detected precessing frequency in the present case is $1970 \pm 20 \mathrm{~Hz}$ which is interestingly similar to the nonreacting case. The lifted flame thus seems to correspond to a "natural" premixed-like flame stabilization inside the chamber.

In the upstream part of the bottom half of Fig. 3, liquid fuel and flame signal overlap. This is actually an artefact of the averaging procedure: the lifted state is characterized by a strong thermo-acoustic instability around $330 \mathrm{~Hz}$, related to the quarter-wave mode of the combustion chamber. This instability generates a high sound pressure level in the chamber, around $145 \mathrm{~dB}$. In order to better understand the relative motions of the liquid fuel and flame during one acoustic cycle, synchronized post-treatments must be performed.

To analyze the acoustic cycle from the fuel spray and flame points of view, the response of the associated diagnostics (Mie scattering and $\mathrm{CH}^{*}$ chemiluminescence) to the thermo-acoustic instability must be extracted and synchronized. These two steps are usually performed by phase averaging methods with the help of a reference signal that shows a strong response to the probed phenomenon. In the framework of this study, a different technique based on Dynamic Mode Decomposition (DMD, [8]) is used. For each diagnostic, a data matrix is created with 500 snapshots containing the data captured by the camera as well as the simultaneously recorded microphone signal (multi-variable DMD, as defined in [9]). Both matrices are processed separately with a DMD algorithm to obtain the mode associated with a $330 \mathrm{~Hz}$ oscillation. The part of the modes corresponding to the microphone data are used as a reference signal to synchronize the phases of the Mie scattering and the chemiluminescence modes.

In Fig. 4, the Mie scattering DMD mode at the instability frequency shows that the spray reacts to the thermo-acoustic instability in a globally bulk fashion and thus generates equivalence ratio fluctuations that can impact the flame $[10,11]$. In the same images, the DMD mode at $330 \mathrm{~Hz}$ for the $\mathrm{CH}^{*}$ chemiluminescence diagnostic also shows a global response of the flame to the acoustic wave, especially regarding its presence in the upstream part of the chamber. Interestingly, the flame and the liquid fuel seem to show opposite responses: when the chemiluminescence signal is high in the beginning of the chamber $\left(90-180^{\circ}\right)$, the fuel spray signal is low and conversely. There is thus a delay between the liquid fuel feeding and the flame reaction. The mean value of the phase difference between the Mie scattering and the chemiluminescence signals is $-125^{\circ}$ with a $95 \%$ confidence interval of $\pm 4^{\circ}$. This means that the chemiluminescence signal lags $1.04 \pm 0.03 \mathrm{~ms}$ behind the Mie scattering signal.

The time delay between the liquid spray and the flame probably comes from the combination of two phenomena: the droplets must vaporize and then burn before the peak in chemiluminescence is observed. At $1200 \mathrm{~K}$ (which is the combustor outlet temperature), the ignition delay time for kerosene is under $0.3 \mathrm{~ms}$ [12]. It is expected to be even lower here since the temperature assumption is conservative and hot burnt gas recirculation promotes the reaction [13]. Most of the delay time must thus come from the vaporization of the droplets. For the current operating point, the mass median diameter for the spray is $23 \mu \mathrm{m}$, meaning that half of the liquid fuel mass is composed of smaller droplets and half of bigger droplets. A dodecane droplet with such a diameter takes between 0.5 and $2 \mathrm{~ms}$ to vaporize in atmospheres between $433 \mathrm{~K}$ (preheating temperature) and $1800 \mathrm{~K}$ (adiabatic temperature for the present case). Most of the $1.04 \mathrm{~ms}$ delay can thus be attributed to fuel vaporization and since this delay also represents a part of the oscillations feedback mechanism, changes in fuel or spray properties can have a strong impact on the stability of the system.

\section{Triggered transition}

In the present section, a way to switch from the $s V$ state to the lifted state is investigated. The siren, described in section 2 is operated at a fixed frequency of $290 \mathrm{~Hz}$ and the amplitude of the modulations is slowly 

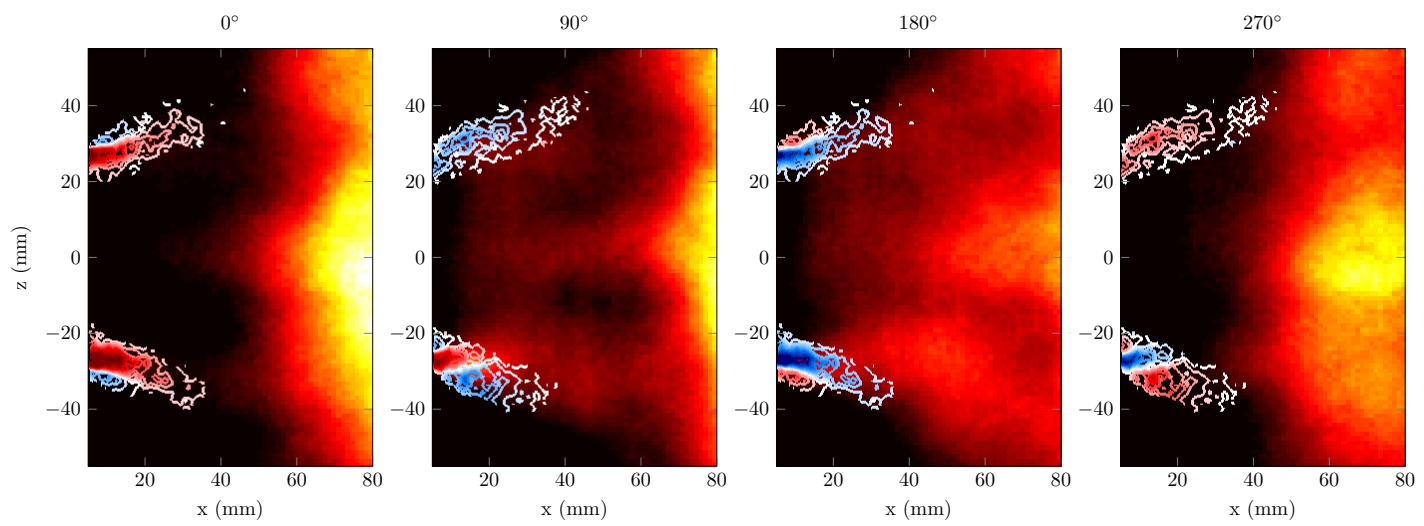

Figure 4: Synchronized DMD modes at the thermo-acoustic instability frequency for four phase angles. The liquid fuel spray from Mie scattering is in blue (signal below average), white and red (signal above average). The mode from $\mathrm{CH}^{*}$ chemiluminescence is added to the average image to highlight the motion of the flame in shades of red, orange and yellow.

increased until the flame switches to the lifted state with its associated thermo-acoustic instability.

In order to study this triggered lift of the flame, the time-frequency content of two signals is analyzed. The first signal comes from the internal Mie scattering visualization and responds well to the PVC. It enables to detect the switch between the two states from the internal aerodynamical point of view as both states are characterized by different PVC frequencies. The second signal comes from the microphone and enables to detect the onset of the $330 \mathrm{~Hz}$ thermo-acoustic instability that characterizes the lifted state. Continuous wavelet transform [14] is used for the time-frequency post-processing and the resulting scalograms can be seen in Fig. 5 .

The mother wavelet is the complex Morlet one. The scales are chosen to provide a scalogram coefficient every $2 \mathrm{~Hz}$. Since we are interested here in the temporal detection of a phenomenon, the bandwidth parameter of the wavelets is chosen so that they give the same $\pm 0.02 \mathrm{~s}$ precision at $2000 \mathrm{~Hz}$ for the internal Mie scalogram and at $310 \mathrm{~Hz}$ for the microphone one. The frequency resolution is thus different between the two images in Fig. 5 but the temporal resolution is the same.

At the top of Fig 5, it can be seen that the PVC frequency is above $2000 \mathrm{~Hz}$ in the beginning and between $1950 \mathrm{~Hz}$ and $2000 \mathrm{~Hz}$ in the end. The switch from the $s V$ state (high PVC frequency) to the lifted state (low PVC frequency) can clearly be identified as an abrupt frequency drop around $0.15 \mathrm{~s}$. This change corresponds to a modification of the air flow inside the injector.

In the scalogram for the microphone signal (bottom of Fig. 5), the effect of the siren can easily be detected around $290 \mathrm{~Hz}$. In the end, a second structure can be observed near $330 \mathrm{~Hz}$ : it corresponds to the thermoacoustic instability associated with the lifted flame. The onset of this instability seems to be after $0.2 \mathrm{~s}$, that is, after the switch to the lifted state from the aerodynamical point of view.

Figure 5 thus shows that the lift of the flame happens before the onset of the thermo-acoustic instability which is then a mere consequence of the change of the flame and flow structures. This switch probably happens because the strong air flow modulations created by the siren blow the internal flame anchored on the pilot nozzle off [15]. The $s V$ state is thus extremely sensitive to external perturbations since the flame remains in the lifted state even when the siren is stopped after the switch.

\section{Conclusion}

In a staged gas turbine model combustor, a bistable behaviour is observed when most of the liquid fuel is injected through a multipoint stage. The occurrence of the states depends on the burner history as well as on potential external perturbations.

The $s V$ state corresponds to a case where the air flow and the spray seem confined, probably because of an internal flame located near the pilot nozzle that shapes the air flow structure. It is characterized by a marked Precessing Vortex Core with a frequency above $2000 \mathrm{~Hz}$. Its moderate and broadband acoustic activity as well as its seemingly well mixed combustion lead to think that this state has interesting properties for industrial applications. It is however shown that it is sensitive to perturbations. The use of air flow rate modulations at $290 \mathrm{~Hz}$ can indeed lead to the extinction of the internal flame, making the burner switch to the lifted state.

The lifted state is globally similar to the non-reacting conditions from the air flow point of view. The flame is 

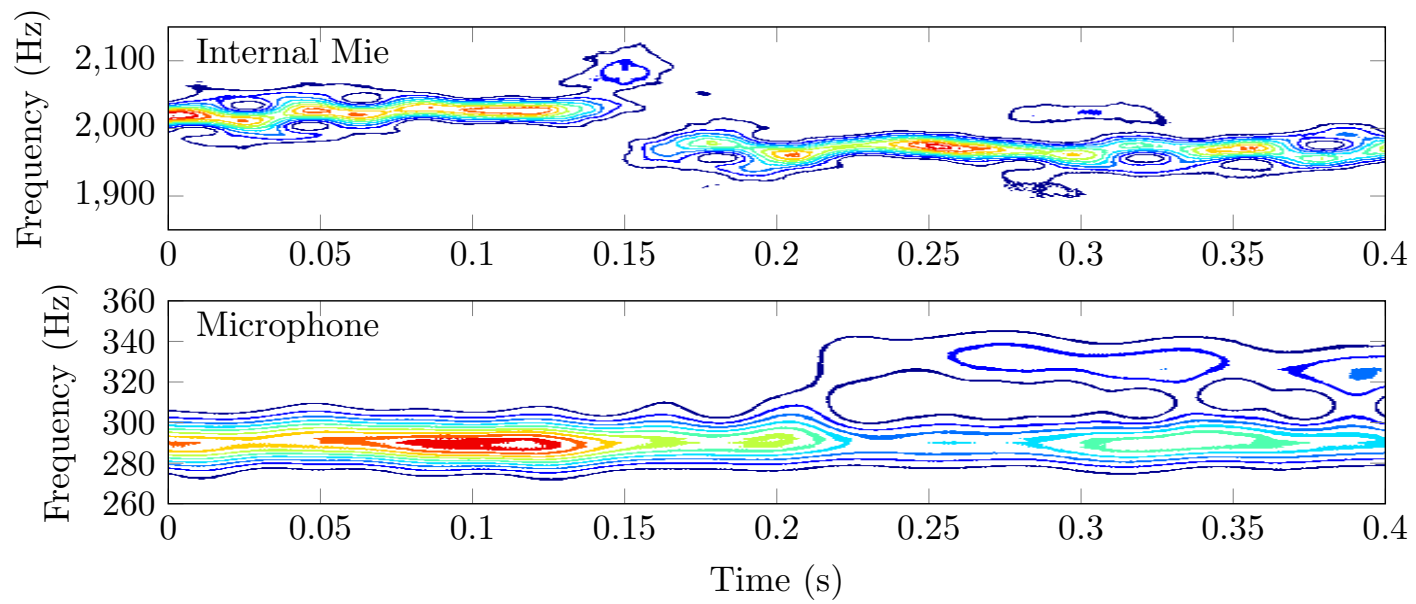

Figure 5: Scalograms of two signals during the siren-induced lift of the flame. Top: internal Mie scattering signal around the PVC frequency. Bottom: microphone signal around the thermo-acoustic instability frequency. The contours represent levels corresponding to multiples of $10 \%$ of the maximum.

detached from the injector and stabilizes in the chamber in a relatively spread fashion. From a dynamical point of view, this state is characterized by a PVC close to the one observed in non-reacting conditions: not so strong and around $1970 \mathrm{~Hz}$. The most interesting feature of this state is the fact that it triggers a thermo-acoustic instability based on the quarter-wave mode of the combustion chamber. This can be observed through the oscillations of the liquid fuel spray and the flame with a delay. The value of this delay is mainly piloted by the fuel vaporization time, leading to think that a change in fuel or spray properties can have a dramatic impact on the instability.

To gather more information on these phenomena, 3D measurements enabling to access the out-of-plane behaviour would be fruitful as well as fuel vapour phase measurements to obtain the missing link between the spray and the flame.

\section{Acknowledgements}

The authors would like to gratefully acknowledge Safran/Snecma for supporting this study. We also thank Fabrice Giuliani of CBOne for designing, making and helping set up the siren device as well as Philippe Scouflaire for his help with the diagnostics hardware.

\section{References}

[1] S. Candel, Proceedings of the Combustion Institute 29 (2002) $1-28$.

[2] Y. Huang, V. Yang, Progress in Energy and Combustion Science 35 (2009) 293-364.
[3] W. Krebs, P. Flohr, B. Prade, S. Hoffmann, Combustion Science and Technology 174 (2002) 99-128.

[4] S. Hermeth, G. Staffelbach, L. Y. Gicquel, V. Anisimov, C. Cirigliano, T. Poinsot, Combustion and Flame 161 (2014) 184-196.

[5] T. Providakis, L. Zimmer, P. Scouflaire, S. Ducruix, Journal of Engineering for Gas Turbines and Power 134 (2012).

[6] A. Renaud, S. Ducruix, P. Scouflaire, L. Zimmer, Proceedings of the Combustion Institute 35 (2015) 3365-3372.

[7] F. Giuliani, A. Lang, K. J. Gradl, P. Siebenhofer, J. Fritzer, Journal of Engineering for Gas Turbines and Power 134 (2012).

[8] P. J. Schmid, Journal of Fluid Mechanics 656 (2010) 5-28.

[9] F. Richecoeur, L. Hakim, A. Renaud, L. Zimmer, Proceedings of the Summer Program (2012) 459.

[10] T. Lieuwen, B. T. Zinn, Symposium (International) on Combustion 27 (1998) 1809-1816.

[11] T. Sattelmayer, Journal of Engineering for Gas Turbines and Power 125 (2003) 11.

[12] B. Franzelli, E. Riber, M. Sanjosé, T. Poinsot, Combustion and Flame 157 (2010) 1364-1373.

[13] R. Sadanandan, M. Stöhr, W. Meier, Applied Physics B 90 (2008) 609-618.

[14] A. Grossmann, R. Kronland-Martinet, J. Morlet, in: Wavelets, Springer, 1989, pp. 2-20.

[15] Y. Hardalupas, A. Selbach, J. H. Whitelaw, in: Laser techniques applied to fluid mechanics, Springer, 2000, pp. 403-416. 